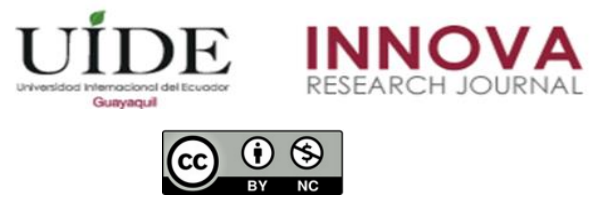

INNOVA Research Journal, ISSN 2477-9024

(Septiembre-Diciembre 2019). Vol. 4, No.3.1 pp. 30-41

DOI: https://doi.org/10.33890/innova.v4.n3.1.2019.1175

URL: http://revistas.uide.edu.ec/index.php/innova/index

Correo: innova@uide.edu.ec

\title{
Estrategias financieras para incrementar la rentabilidad. Caso: Empresa Indema Perú S.A.C
}

\section{Financial strategies to increase profitability. Case: Empresa Indema Perú S.A.C}

\author{
Zurita Farceque Trinidad \\ Pucutay Perez Jen Alexis \\ Córdova Jaime Iduvina \\ León Sánchez Liliana Yolanda \\ Universidad Norbert Wiener, Perú
}

Autor para correspondencia: trinidadzuritaf1406@gmail.com; jen.pp2791@gmail.com; iducj26@gmail.com; ls.lily.20@gmail.com

Fecha de recepción: 27 de agosto de 2019 - Fecha de aceptación: 21 de octubre de 2019

\section{Resumen}

El objetivo de la investigación fue proponer la implementación de estrategias financieras para incrementar la rentabilidad de la empresa Indema Perú S.A.C. Las principales causas del estancamiento en el desarrollo económico empresarial están constituidas por el elevado costo de ventas y la falta de control en los gastos que afectan de manera directa a la utilidad, reflejándose en los respectivos índices. La investigación fue de enfoque mixto, método analítico e inductivo, en la recopilación de datos se aplicó la guía de entrevista a tres unidades informantes que fueron el gerente general, la contadora y el jefe de finanzas, también se aplicó la ficha de análisis documental, los datos se triangularon con el software Atlas. Ti. Los resultados evidenciaron que la rentabilidad sobre los activos disminuyó año tras año, para el año 2016 la empresa tuvo un rendimiento de $0.86 \%$ sobre la inversión, en el 2017, generó pérdidas; sin embargo, para el año 2018, obtuvo un $0.08 \%$ de rendimiento sobre la inversión. Los factores que influyen de manera directa en la rentabilidad es el alto costo de ventas, que en el 2018 representó un $85 \%$ del nivel de ventas y se espera reducir al 70\% en los próximos 5 años.

Palabras claves: rentabilidad; estrategias financieras; gestión financiera; liquidez; crecimiento económico

\footnotetext{
Abstract

The objective of the investigation was to propose the implementation of financial strategies to increase the profitability of the company Indema Perú S.A.C. The main causes of stagnation in business economic development are constituted by the high cost of sales and the lack of control over expenses that directly affect profit, reflected in the respective indices. The investigation was of mixed approach, analytical and inductive method, in the data collection the interview guide was applied to three informant units that were the general manager, the accountant and the head of finance, the documentary analysis sheet was also applied, the data was triangulated with the Atlas software. You. The results showed that the profitability on the assets decreased year after
} 
year, for the year 2016 the company had a yield of $0.86 \%$ on the investment, in 2017 , it generated losses; however, for the year 2018, it obtained a $0.08 \%$ return on investment. The factors that directly influence profitability is the high cost of sales, which in 2018 represented $85 \%$ of the sales level and is expected to reduce to $70 \%$ in the next 5 years.

Key words: profitability; financial strategies; financial management; liquidity; economic growth

\section{Introducción}

El problema de los bajos resultados en la rentabilidad de las empresas de servicios habitualmente es por la falta de creatividad gerencial, cuyas consecuencias son las pérdidas económicas, los inversionistas buscan oportunidades en países emergentes con el fin de proteger los intereses de su patrimonio, uno de ellos es Brasil, por los recursos naturales y el número de sus pobladores, del mismo modo, China y Rusia que se diferencian por su formidable desarrollo económico. Respecto al crecimiento monetario sustancial para el progreso del Estado, es necesario cubrir las necesidades de los diversos tipos de mercado competitivo, por ello, el propósito de las organizaciones es plantear estrategias para incrementar la rentabilidad, la cual debe enfocarse en técnicas que ayuden a la compañía acceder a líneas de créditos, con la finalidad de posicionarse mediante una adecuada gestión económica - financiera (Daza, 2016; Puente y Viñán, 2017; Cantero \& Leyva, 2016).

La perspectiva de la economía peruana es incrementar en un 4\% el desarrollo económico a partir del año 2019 (Ministerio de Economía y Finanzas-MEF, 2018), dando posibilidades de crecimiento y expansión continua a las empresas, no obstante, la falta de aplicación de estrategias económicas y financieras por parte de la compañía de servicios "Indema Perú S.A.C.”, conduce a la reducción de la rentabilidad en un determinado periodo, originando en los accionistas la preocupación de sus inversiones e inseguridad de continuar apostando por la misma. En este sentido, las estrategias direccionarán los objetivos planteados por la entidad, permitiendo la obtención de la utilidad, tal como lo indicó la Cámara de Comercio de Lima (CCL), que el sector de servicios aporta al producto bruto interno (PBI) (MEF, 2018; Miranda, 2017; Cantero \& Leyva, 2016).

La rentabilidad en términos generales es la capacidad de ingresos que una empresa genera, mediante recursos propios y/o de terceros, la misma que admite minimizar la incertidumbre ante cualquier situación que se prolongue. La gerencia debe de tomar decisiones referentes a la situación económica en la que se encuentra la empresa de servicios, analizando los índices financieros con el apoyo del desarrollo de los ratios de rentabilidad; en el cual los porcentajes obtenidos en el año 2018 referente al ROA (rentabilidad sobre los activos) fue de $0.36 \%$ y en el ROE (Rentabilidad sobre el patrimonio) se tuvo un $0.27 \%$, los cuales no son considerados factibles para el desarrollo productivo de la entidad, por esta razón se espera que estos mejoren en los próximos cinco años (Contreras, Stein y Vecino, 2015; Villada, López y Muñoz, 2018; Elías y Díaz, 2015).

Los factores que intervienen en los bajos niveles de rentabilidad son los elevados costos de producción, los cuales están relacionados con la mano de obra, materia prima y otros costos indirectos de fabricación, así también corresponde a los altos costos de los recursos humanos y tecnológicos. Para dar solución a la problemática se propone: Incrementar los ingresos, mediante 
estrategias de venta con el fin de captar de nuevos clientes, de la misma manera aplicar estrategias de marketing en base a las 4P: a) Producto, la calidad que busca todo emprendedor para ofrecer a sus consumidores, con la finalidad de satisfacer sus necesidades; b) Plaza, la captación de los nichos de mercado para comercializar los bienes; c) Precio, el valor final asignado a los productos comercializados en el mercado; d) Promoción, el punto de inicio para lograr afianzar los servicios con la intención de dar a conocer las ofertas al mercado competitivo (Morelos y Nuñéz, 2017; Quinde \& Ramos, 2018; Villegas, Hernández, \& Salazar, 2017; Ferré y Ferré, 1996; Kotler y Armstrong, 2003; Meoño \& Escoto, 2006; Porter, 2005; Sallenave, 2002).

El financiamiento es considerado una herramienta fundamental para aumentar los ingresos, además de fortalecer el desempeño económico dentro del mercado local, así mismo, los recursos que dispone la empresa "Indema Perú S.A.C.", no son suficientes para hacer frente a sus obligaciones a corto plazo, a causa de ello, se plantea incrementar la liquidez a través de la actualización de los créditos y cobranzas, permitiendo conocer la situación real de los clientes (riesgo crediticio), por otra parte, implementar en la gestión financiera como los descuentos por pronto pago e interés por morosidad (Vera, Melgarejo y Mora, 2014); vinculando así la teoría administrativa financiera que permitirá lograr las metas gerenciales, optimizando el progreso económico mediante la diversificación del riesgo financiero para maximizar la rentabilidad (Meoño y Escoto, 2006; Agüero,2007; Hernández, Treviño y López 2017).

El óptimo manejo de la gestión de las inversiones y gastos operativos conllevan a conseguir un resultado atractivo y acceder a instrumentos financieros; por ende es necesario contar con un presupuesto proyectado que equilibre los gastos e ingresos, contribuyendo con el ahorro y manejo eficiente del efectivo, el incremento de la liquidez aumentará las posibilidades de mejora en la rentabilidad para asumir las obligaciones tributarias (Vera, Melgarejo, \& Mora, 2014; Pérez, 2013), teniendo de base la teoría de la decisión que permite la planificación de estrategias de la situación económica y financiera de la empresa para dar solución y perfeccionar los resultados en un determinado tiempo (Chiavenato, 2006). De la misma manera con la teoría económica de la empresa para analizar, aclarar la situación actual de la organización y la conducta humana con la finalidad de aumentar la eficacia (García,1994; Folke, 1997).

\section{Materiales y métodos}

El estudio de investigación proyectiva fue elaborado mediante el enfoque mixto, nivel comprensivo, método analítico e inductivo, la categoría principal es la rentabilidad, las subcategorías fueron los ratios de rentabilidad, factores de rentabilidad, financiamiento y gestión económica - financiera. Para la recopilación de datos se ejecutó a través de entrevistas a cuatro unidades informantes que fueron el gerente general, la contadora, la gerente de logística y el jefe de finanzas, asimismo se aplicó la ficha de análisis documental a los estados financieros, de los cuales se interpretaron y analizaron de manera vertical y horizontal, además se calculó los ratios financieros de rentabilidad, finalmente para el diagnóstico se realizó la triangulación de datos con el apoyo del software Atlas. Ti. 


\section{Resultados}

En la interpretación de los ratios financieros de rentabilidad según el análisis cuantitativo, se determinó que el ROA (Return on assets) disminuyó en los últimos tres años (en el año 2016, 0.86\%, año 2017, 0\% y en el año 2018, 0.36\%), por lo tanto, el rendimiento que generó la empresa producto de su inversión se redujo anualmente en un $0.40 \%$ aproximadamente.

Respecto al indicador ROE (Return on equity), los resultados disminuyeron a consecuencia de los altos costos de servicios, para el año 2016 fue de $2.25 \%$, en el año 2017 de $0 \%$ y para el año 2018 de $0.27 \%$, por ende, los resultados no fueron los deseados por los accionistas de la entidad.

Referente al nivel de ventas entre los periodos del 2016 al 2017 los resultados crecieron en un 175\%, mientras que en los periodo del 2017 al 2018 hubo una reducción de un 30\%, los mismos que fueron ocasionados por la inadecuada gestión del financiamiento, elevado costo de ventas, que en el periodo 2016 fue de $70 \%$, para el año 2017 de $110 \%$ y para el 2018 de $85 \%$, así como también la falta de planeación estratégica el cual se vio reflejado directamente en los resultados finales de los estados financieros.

En relación a los factores que influyen en la disminución de la rentabilidad como 1 escases del apalancamiento, innecesarios costos de mano de obra, materiales y gastos indirectos, en el último año ha mejorado, sin embargo, este contexto requiere de un control firme, dado que existe una relación directa entre el manejo incorrecto de la producción y la administración para la toma de decisiones.

Para mejorar la rentabilidad de la empresa se elaboró estrategias financieras estableciendo los siguientes objetivos: Incrementar las ventas, reorganizar los costos de acuerdo a las necesidades por cada proyecto e incrementar la liquidez, el cual se refleja en la figura número 1:

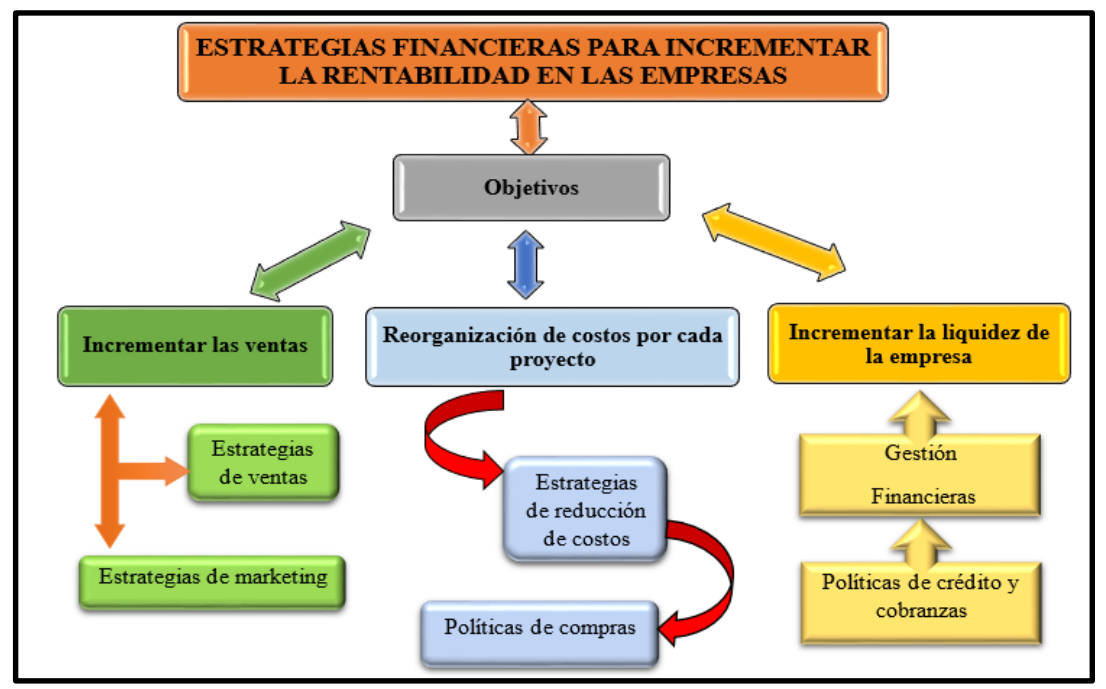

Figura 1. Estrategias financieras para incrementar la rentabilidad. Fuente elaboración propia. 


\section{Objetivo 1: Incrementar las ventas}

El nivel de los ingresos ordinarios de la compañía en estudio se redujo en un $30 \%$ en el último periodo, del cual se estima prosperar en un $12 \%$ en los próximos cinco años. Para lograr el propósito se plantea estrategias de ventas y de marketing, que permitirá evaluar el entorno del mercado competitivo.

\section{Estrategias de marketing}

La publicidad y redes de mercadeo se han convertido en una fuente necesaria e indispensable para expandir y dar a conocer el servicio que la compañía ofrece, teniendo como finalidad el incremento de las ganancias que se verán reflejados en el estado de resultados. Para ello se propone efectuar una publicidad a través de fuentes de mayor utilización de hoy en día, como las redes sociales, la publicidad impresa (tarjetas, afiches), correos corporativos a sus clientes que más aportan a sus ingresos de la empresa y la publicidad boca a boca que permitirá mediante terceros dar a conocer el servicio que ofrece la entidad (Ferré, J.M, \& Ferré, J. 1996; Puente y Viñán, 2017). Como se muestra en la figura número 2:

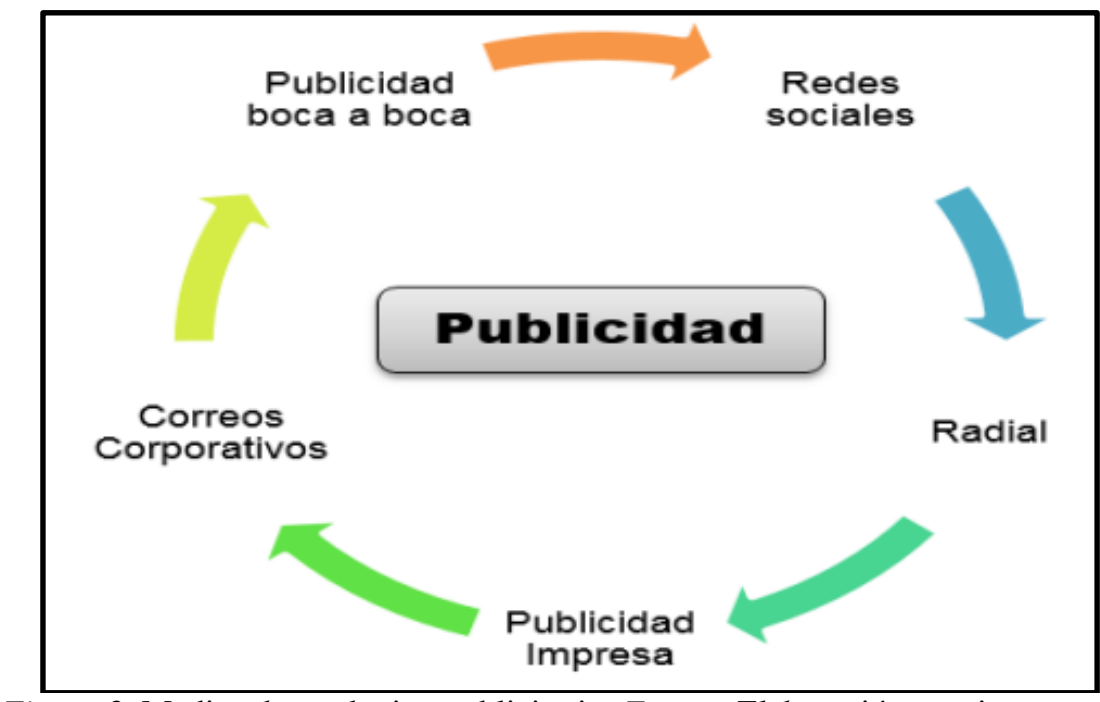

Figura 2. Medios de marketing publicitario. Fuente: Elaboración propia.

\section{Mercadotecnia}

La fabricación de los bienes de la empresa mayormente es ejecutada en el exterior, por lo que esto genera costos. A consecuencia de este, se prevé realizar una comparación de los precios que se fijan en el mercado de acuerdo a las diferentes empresas, solicitando que el proveedor emita una cotización de los productos posibles a ser adquiridos, esto ayudara a poder evaluar y analizar. De modo que la entidad optará por elegir el bien a menor precio con la finalidad de obtener mayor rentabilidad, ante eso se tendrá en cuenta las 4p (producto, plaza, precio, promoción) (Morelos y Nuñez, 2017; Porter, 2005). 
Producto: Al otorgar la calidad del producto al usuario, ante todo teniendo en cuenta la buena la presentación, la marca con la que se identifica, el diseño del producto, con el fin de satisfacer las necesidades del cliente.

Plaza: Evaluamos los nichos de mercado para identificar hacia donde van hacer dirigidos los bienes y servicios que se ofrecerá al cliente, de forma que logremos el posicionamiento en el mercado local, generando la seguridad de la salida del bien.

Precio: Para asignar el precio de los productos y servicios, se solicitará a los proveedores que coticen ciertos productos y/o servicios con la finalidad de aplicar el precio correspondiente a cada bien y/o servició que se le ofrece al cliente, siendo el punto clave del éxito del negocio.

Promoción: Los bienes y/o servicios que brinda la empresa, para dar a conocer las promociones que otorgara, se realizó a través del marketing, debido a que esto genera tener un alto índice de ventas, atrayendo la atención de los clientes y mejorar la imagen de la entidad. Los cuales se identifican en la figura número 3:

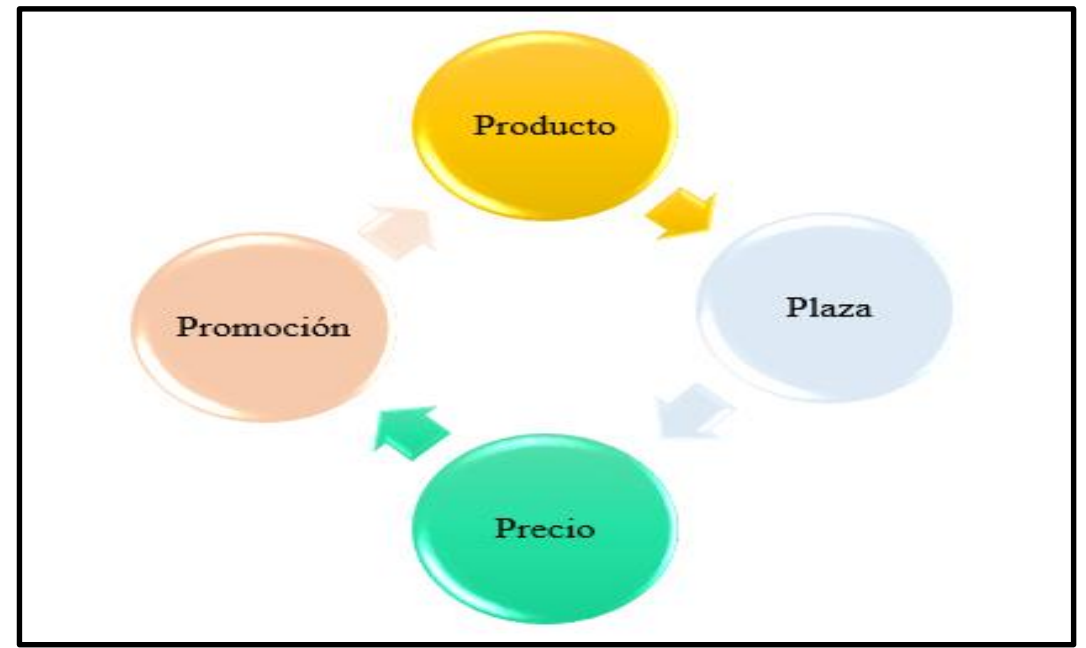

Figura 3. Procesos de estudio de mercado. Fuente: elaboración propia.

\section{Estrategias de venta}

Dentro de las estrategias de venta, primero se analizará al mercado competitivo recolectando información acerca de la oferta, la demanda y los precios con el propósito de encaminar a un futuro mejor, por ende, tomar decisiones acertadas que favorezcan el crecimiento de los ingresos de la entidad. Así mismo dichas estrategias conllevará a captar nuevos clientes que generen un alto nivel comercial en un mediano plazo (Morelos y Nuñéz, 2017; Quinde \& Ramos, 2018).

\section{Captación de nuevos clientes}

Es un reto para los sectores de conseguir nuevos clientes, con el fin de obtener altos niveles de ingresos. Para ello, se creó un plan de operaciones que inicia con la definición del perfil del usuario, utilizando fuentes de contactos, clasificación según su potencial económica, 
luego conseguir una entrevista para continuar con los procesos hasta culminar con la aceptación del proyecto a realizar. Como señala la figura número 4:

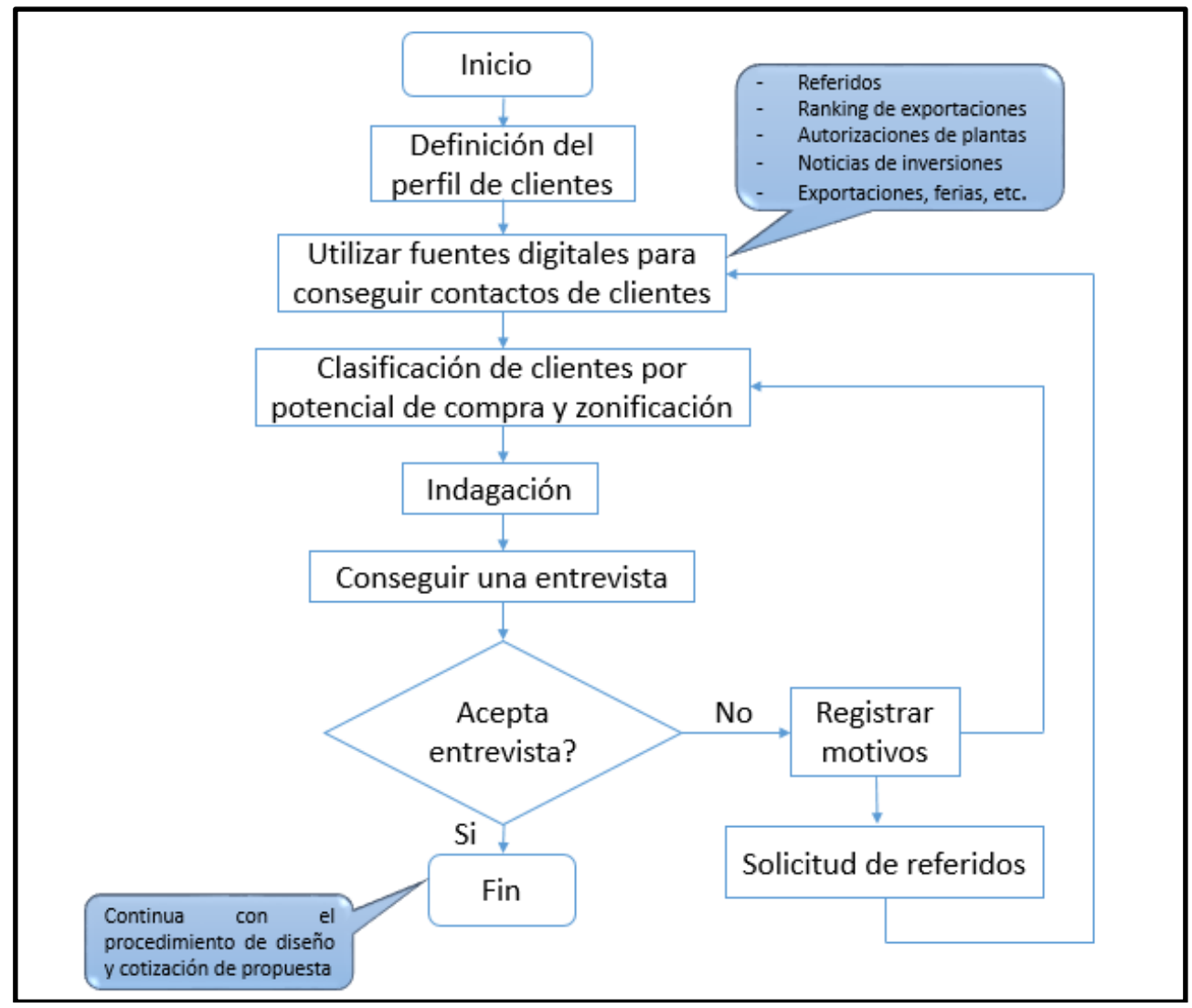

Figura 4. Procedimientos para conseguir nuevos clientes. Fuente: elaboración propia.

\section{Objetivo 2: Reorganización de costos por cada proyecto}

La restructuración de la gestión de proyectos permitirá reducir los costos indebidos de la empresa, por lo que se planteó estrategias con el propósito de aumentar la rentabilidad en los próximos años. Actualmente el costo de ventas está en un $85 \%$ y se espera reducir un 3\% anual durante los próximos años, asimismo lo gastos operativos que hoy representan un $12 \%$ se espera reducirlos a $9 \%$.

\section{Gestión eficiente del inventario}

La gestión eficiente en los inventarios permitirá que las operaciones simbolicen una ventaja competitiva para el mercado, a la vez tomar decisiones adecuadas. Por lo tanto, se establecerá nuevas tácticas en la adquisición de bienes, aplicando la tecnología sistemática con novedosas políticas de compra que perfeccionen el control interno del área de logística (Meoño y Escoto, 2006; Hernández, Treviño y López, 2017). 


\section{Procesos de compra}

Añadir un plan adecuado de compras, facilita a que los inventarios sean controlados oportunamente y no genere costos adicionales. Al perfeccionar políticas de compras, las actividades que realicen las organizaciones serán eficientes.

Para la cotización de una adquisición, se debe de seguir los siguientes pasos: Primero crear una hoja u formato de requerimientos, controlar las solicitudes mediante un cuadro comparativo, seguido ejecutar la orden la compra para que sea aprobado, verificar el ingreso del producto y por último coordinar la forma de cancelación de la factura. Como se observa en la figura número 5:

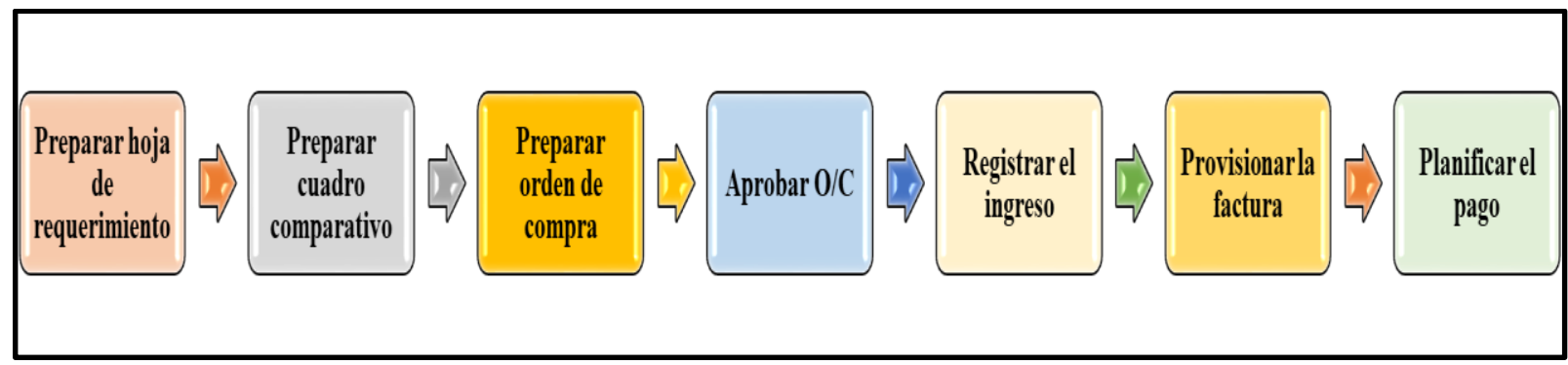

Figura 5. Procesos para mejorar las cotizaciones de las compras. Fuente. Elaboración propia.

\section{Implementar un software}

Crear un ERP (Enterprise resource planning) conlleva a tener una comunicación en línea optima, donde la información ingresada por los responsables sea vinculada de manera directa con todas las áreas, siendo una modalidad eficaz para minimizar tiempo y desembolsos innecesarios. El cual se señala en la figura número 6:

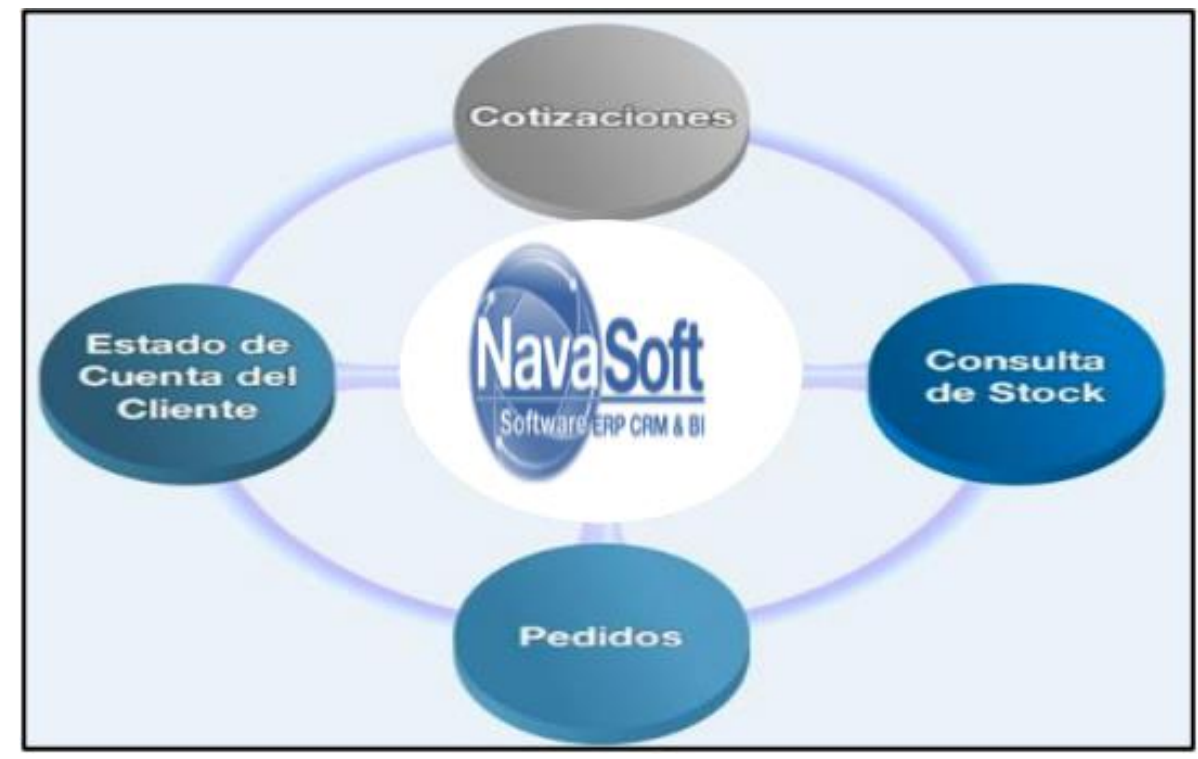

Figura 6. Implementación de software para la mejora de las compras. Fuente: Elaboración propia. 


\section{Objetivo 3: Incrementar la liquidez.}

Muchas empresas no disponen de efectivo suficiente para cumplir con sus obligaciones de corto plazo, por eso es preciso tener bien establecidas y definidas las políticas de créditos y cobranzas que permitan cumplir con los plazos de cobro y pago establecidos (Folke, 1997; García, 1994).

Se estima tener un punto neutro entre el activo exigible y las obligaciones a corto plazo, por lo que las cuentas por cobrar a 108 días se cobren a 60 días; mientras que las cuentas por pagar que hoy son a 51 días se paguen a 90 días, siendo un beneficio para elevar el activo disponible (Vera, Melgarejo y Mora, 2014).

\section{Políticas de créditos y cobranzas}

Los lineamientos de las políticas de créditos y cobranzas son procedimientos que deben seguir las entidades con fines de lucro con el propósito de reducir las facturas vencidas, a la vez amortizar los pasivos a corto plazo, mediante una actualización de la gestión interna del área de tesorería mediante procesos continuos para el otorgamiento de los créditos con los nuevos clientes (Vera, Melgarejo, \& Mora, 2014; Pérez, 2013). Como se numera en la figura 7:

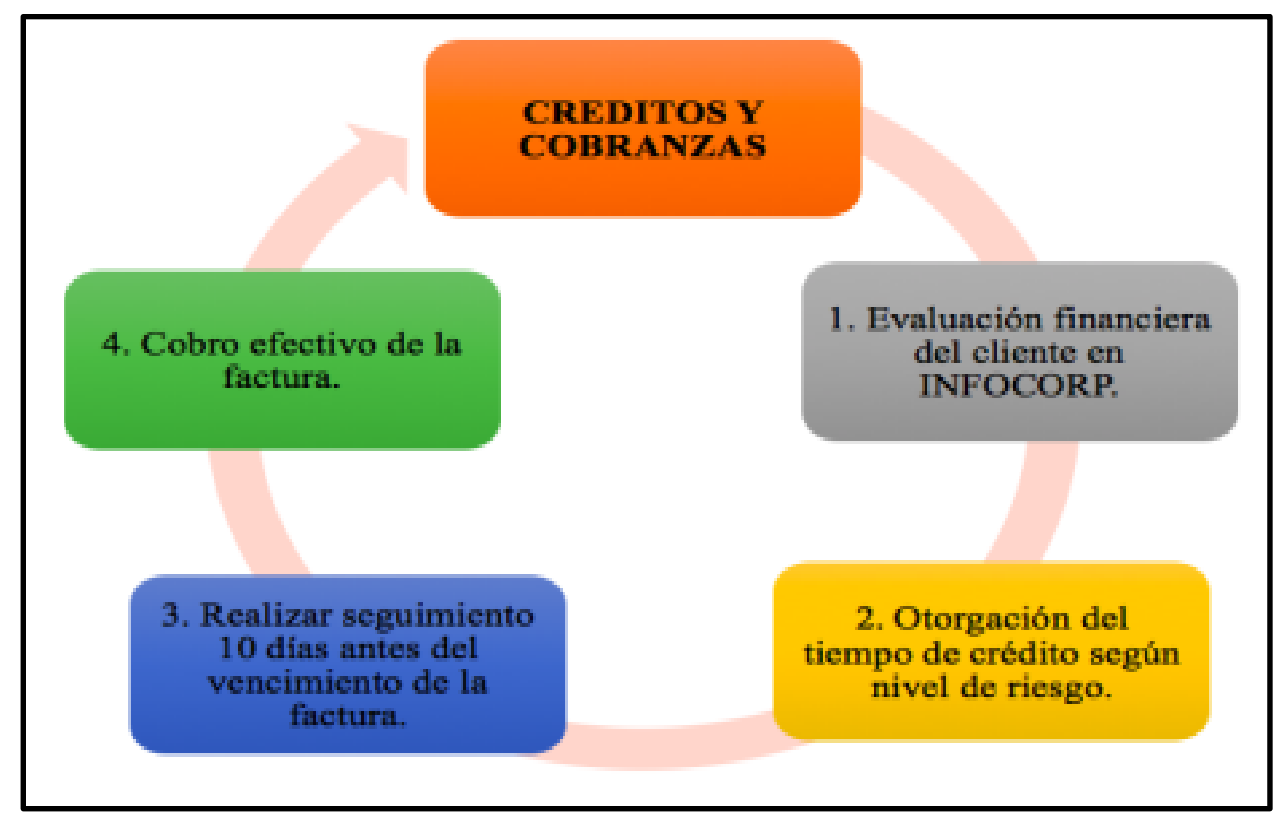

Figura 7. Solución para mejorar las políticas de créditos y cobranzas. Fuente: Elaboración propia.

\section{Gestión Financiera}

La gestión financiera permitirá mejorar la rentabilidad de la empresa, administrar los capitales con los que dispone para cubrir las obligaciones con terceros, de esta manera contar con un control adecuado de los accesos y partidas que genere la entidad (Meoño y Escoto, 2006; Agüero, 2007). 


\section{Descuento por pronto pago}

El pronto pago tiene como objetivo estimular a los clientes a optar por el pago de sus obligaciones antes de su vencimiento, así mismo será un beneficio directo y paralelo para ambas partes vinculadas en cuanto al disponible. Para alcanzar la meta planteada de reducir el porcentaje de clientes morosos, se plantea un descuento del $4 \%$ a la factura emitida

\section{Intereses moratorios}

El interés moratorio es un porcentaje que se cobra por el incumplimiento del plazo señalado en el contrato, las mismas que vienen a ser una indemnización mínima para el acreedor hasta un 4\%, todo ello con el propósito de eliminar la morosidad y disminuir las cuentas incobrables y estas a su vez permitirá incrementar la liquidez de la empresa.

\section{Conclusiones}

Las estrategias financieras son instrumentos esenciales para extender las ganancias de la empresa (Ahumada \& Perusquilla, 2016), así mismo contribuyen a apoderarse de una colocación más competitiva en el sector, sin embargo, dichas maniobras no son aprovechadas de manera adecuada, en consecuencia, no permite generar ganancias en un periodo determinado (Chiavenato, 2006).

De acuerdo al análisis de los ratios financieros de rentabilidad, miden los beneficios que conciben las entidades mediante sus activos y el patrimonio. En conclusión, se determinó que la empresa no cuenta con una utilidad adecuada, la misma que no contribuye al continuo crecimiento económico de manera óptima (Cantero \& Leyva, 2016).

El nivel de ventas disminuyó, por ende, no permitió el desarrolle con normalidad las actividades de la organización, dado que conllevó a solicitar un financiamiento, el mismo que generará deudas a largo plazo, las cuales serán canceladas según el cronograma instruido con el apoyo del cumplimiento de las estrategias previamente establecidas (Alva, 2017; García,1994).

Respecto a los altos costos de ventas se reorganizarán el manejo de los costos mediante estrategias de gestión en los inventarios e implementación del ERP, reduciendo así los costos y/o gastos innecesarios que la empresa suele realizar para mantener la liquidez estable de la empresa (Ahumada \& Perusquilla, 2016).

Para aumentar la liquidez, se establecieron políticas de créditos y cobranzas, gestión financiera a base de descuentos por pronto pago e intereses moratorios a las facturas que se encuentran próximas por vencer y vencidas, con la finalidad de poder administrar los recursos y capitales disponibles (Vera, Melgarejo y Mora, 2014). 


\section{Bibliografía}

Aguero, J. (2007). Teoría de la Administración: Un campo fragmentado y multifacético. Revista cientifica visión de futuro, 7(1). doi:16697634

Ahumada, E., \& Perusquilla, J. (2016). Inteligencia de negocios: Estrategia para el desarrollo de competitividad en empresas de base tecnológica. Revista contaduría y administración (61), 127-158. doi:http://dxdoi.org/10.1016/jcya2015.09.006

Alva, K. (2017). Impacto económico financiero del cumplimiento de normas tributarias en la gestión empresarial. Revista Quipukamayoc, 25(48), 19-25. doi:http://dx.doi.org/10.15381/quipu.v25i48.13987

Banco mundial. (2019). Recuperado el 03 de Julio de 2019, de https://www.bancomundial.org/es/country/peru/overview

Cantero , H., \& Leyva, E. (2016). La rentabilidad económica, un factor para alcanzar la eficiencia empresarial. Revista Ciencias Holguin, 22(4), 1-17.

Chiavenato, I. (2006). Introducción a la teoria general de la administración. México: Mc grawhill.

Contreras, O., Stein, R., \& Vecino, C. (2015). Estrategia de inversión optimizando la relación rentabilidad-riesgo: Evidencia en el mercado accionario colombiano. Revista estudios gerenciales, 31, 383-392. dx.doi.org/10.1016/j.estger.2015.07.005

Daza, J. (2016). Crecimiento y rentabilidad empresarial en el sector industrial brasileño. Revista contaduría y administración, 61, 266-282. doi:http://dx.doi.org/10.1016/j.cya.2015.12.001

Elías, J., \& Díaz, G. (2015). Analísis de los factores que influyen en la toma de decisiones de inversión en portafolios de acciones. Revista publicando, 5(2), 319-329.

Ferré, J.M., \& Ferré, J. (1996). Políticas y estrategias de promoción de ventas y merchandising. Madrid: Ediciones Díaz de Santos S.A.

Folke, K. (1997). Teoría económica. Lima: Centro de investigación de la universidad del pacífico.

García, S. (1994). Teoria económica de la empresa. Mdrid: Ediciones diaz de santos sa.

Kotler, P., \& Armstrong, G. (2003). Fundamentos de marketing. México: Pearson educación.

Kotler, P., \& Armstrong, G. (2017). Fundamentos de marketing (Sexta edicion ed.). Estados Unidos: PEARSON Educacion.

Meoño, M., \& Escoto, R. (2006). Operaciones Bursátiles. Costa Rica: Univeridad Estatal a Distancia.

Miranda, D. (2018). Planeación estratégica para la generación de valor económico agregado. Revista Innova, 3(4), 83-98. doi:https://doi.org/1033890/innova.v3.n4.2018.498

Morelos, J., \& Nuñéz, M. (2017). Productividad de las empresas de la zona extractiva mineraenergética y su incidencia en el desempe no financiero en Colombia. Revista estudios gerenciales, 33, 330-340. doi.org/10.1016/j.estger.2017.11.002

Pérez, J. (2013). Control de la gestion empresarial. Madrid: Esic Editorial.

Porter, M. (2005). Estrategia competitiva: Técnicas para el análisis de los sectores industriales y de la competencia. México: Grupo editorial patria.

Puente, M., \& Viñán, J. (2017). Estrategias de creciomiento empresarial y su incidencia en la rentabilidad empresarial. Observatorio de la economía latinoamericana, 1-10.

Quinde, C., \& Ramos, T. (2018). Valuación y control del inventario y su efecto en la rentabilidad. Revista eumed, 11-33.

Sallenave, J. (2002). La gerencia integral: No le tema a la competencia, témale a la incompetencia. Bogotá: Grupo Editorial Norma.

Esta obra se comparte bajo la licencia Creative Common Atribución-No Comercial 4.0 International (CC BY-NC 4.0) 
Valencia, A. (2016). Impacto financiero, tributario en la gestión económica según el sistema de detracciones. Revista Quipukamayoc, 24(46), 39-46.

Vera, M., Melgarejo, Z., \& Mora, E. (2014). Acceso a la fianciación en PYMES colombianas: Una mirada desde sus indicadores financieros. INNOVA, 24(53), 149-160. doi:http//doi.org/10.15446/innovar.v24n53.43922

Villada, F., López, J., \& Muñoz, N. (2018). Análisis de la relación entre rentabilidad y riesgo en la planeación de las finanzas personales. Scielo, 11(6), 41-52. doi:http://dx.doi.org/10.4067/S0718-50062018000600041

Villegas, E., Hernández, M., \& Salazar, B. (2017). La medición del capital intelectual y su impacto en el rendimiento financiero en empresas del sector industrial en México. Revista Contaduría Administración(62),

184-206. doi:http://dx.doi.org/10.1016/j.cya.2016.10.002 